\title{
Introduction of a Simple and Minimally Invasive Approach for Access to Epidural Space in Cat
}

\author{
Asma Asadian $^{1}{ }^{\circledR}$, Mirsepehr Pedram $^{1,2^{*} \odot}$, Mohamad Mehdi Dehghan ${ }^{1,2}{ }^{\circledR}$, Javad \\ Sadeghinejad $^{\circledR}{ }^{\circledR}$, Majid Masoudifard $^{1}$, Saeide Eftekhari', Roja Ebrahimi ${ }^{1}$ \\ 'Department of Surgery and Radiology, Faculty of Veterinary Medicine, University of Tehran, Tehran, \\ Iran \\ ${ }^{2}$ Institutes of Biomedical Research, University of Tehran, Tehran, Iran \\ ${ }^{3}$ Department of Basic Sciences, Faculty of Veterinary Medicine, University of Tehran, Iran
}

\begin{abstract}
Background: Although various studied have been done to improve therapeutic strategies in resolving spinal cord injuries, robust clinical treatment is not yet available. Developing a standard animal model is essential to achieve effective treatment for such injuries. The present study was performed to introduce a simple, applicable, and minimally invasive approach for access to epidural space in cats.

Methods: We used a percutaneous approach from the lumbosacral junction for stainless steel cannula insertion to the epidural space without fluoroscopy. CT scan, conventional magnetic resonance imaging $(\mathrm{MRI})$, tractography, and behavioral evaluation were used to assess the correct position of the cannula and the neurological condition of the animal.

Results: MRI results showed no significant change in signal intensity index of neural structures under lumbosacral junction. These observations are further supported by tractography, and also behavioral examination during the study.

Conclusion: We found that the percutaneous approach from the lumbosacral junction is a simple and applicable approach that has no side effects and artifact formation in MRI evaluation.

Keywords: Spinal cord injury; Percutaneous approach; Lumbosacral junction; Cat; Minimally invasive.
\end{abstract}

*Correspondence to Mirsepehr Pedram,

Address: Gharib St. Azadi Ave. Tehran, Iran, Postal code: 1419963114 Tel: +989125092937; Email: mpedram@ut.ac.ir

Published online June 30 2021

Citation: Asadian A, Pedram M, Dehghan MM, Sadeghinejad J, Masoudifard M, Eftekhari S, et al. Introduction of a Simple and Minimally Invasive Approach for Access to Epidural Space in Cat. Clin Neurosci J. 2021;8(3):118-123. doi:10.34172/icnj.2021.25.

\section{Introduction}

Spinal cord injuries are an important part of central nervous system disorders which lead to significant neurological disabilities that reduce the quality of life as a result of loss of sensory and motor function. ${ }^{1}$ Efforts to increase knowledge about the pathological mechanisms of spinal cord injuries and the effectiveness of treatment strategies depend on animal modeling. Achieving the appropriate animal model is crucial in confirming research findings. ${ }^{2}$ Significant progress has been made since 1911 when Allen pioneered the first model of spinal cord injury. ${ }^{3}$ Most of the available information on the pathophysiology of spinal cord injuries and related interventions are findings from animal studies. However, the heterogeneity of spinal cord injuries acts as a barrier in translating these findings to similar conditions in human. ${ }^{4}$

Most previous animal models of spinal cord injury required laminectomy and exposure of the spinal cord to induce direct injury, which leaves a surgical artifact that affects imaging data analysis. ${ }^{5}$ Laminectomy itself is used as a treatment for reducing the secondary damage of spinal cord injuries. Soft tissue destruction during this procedure alters the surrounding regenerative environment, which is critical for spinal cord regeneration and therefore leads to undeniable effects on research results. Laminotomy is another approach used to access the epidural space, which is less extensive than a laminectomy. This procedure also causes damage to the vertebrae and surgical intervention is necessary. Therefore, this approach, similar to laminectomy, has disadvantages such as soft tissue damage and the risk of infection. ${ }^{6}$

In this study, we introduce a simple approach to access the epidural space in cats. This closed and percutaneous approach from the lumbar-sacral region reduces the risk of infection and because of the absence of major arteries and nerves in this region, the possibility of serious injury is minimized. Improved magnetic resonance imaging

(C) 2021 The Author(s). This is an open access article distributed under the terms of the Creative Commons Attribution License (http:// creativecommons.org/licenses/by/4.0/), which permits unrestricted use, distribution, and reproduction in any medium, provided the original work is properly cited. 
(MRI) results, is another advantage of this approach. Also, unlike previous methods, it is simple and can be performed with no need for facilities such as fluoroscopy. One of the applications of this approach is balloon catheter placement in the epidural space and minimally invasive spinal cord injury induction models in spinal cord injury studies.

\section{Materials and Methods Animal Model}

All stages of the present study were performed under the National Institutes of Health (NIH) Guide for the Care and Use of Laboratory Animals ${ }^{7}$ and the approval of the Ethics Committee for Animal care of the Faculty of Veterinary Medicine, University of Tehran.

In this study, 15 adult male domestic shorthair cats weighing 3-5 kg were used. All animals were kept in individual cages one week before the start of the study and were evaluated for general and neurological health.

\section{Cannula Insertion}

The area between the seventh lumbar vertebra and the first sacral vertebra was selected to enter the spinal canal and access the epidural space with minimal invasion. Premedication was administrated by intramuscular injection of ketamine $10 \%(5 \mathrm{mg} / 5 \mathrm{~kg}$ ) and medetomidine (10 to $40 \mu \mathrm{g} / \mathrm{kg})$. Intramuscular injection of tramadol ( $2 \mathrm{mg} / \mathrm{kg})$ was also used to induce analgesia. The lumbosacral region was prepared for a standard surgical procedure. Because of the minimally invasive process and the lack of a surgical incision, there was no need to perform the procedure in the operating room, and the whole process was performed on a CT scan table. Siemens (Somatoma, Germany) was used for CT scan. To enter the epidural space, a stainless teel cannula was designed with an inner diameter of 3 $\mathrm{mm}$ and a length of $8 \mathrm{~cm}$, which contained a mandarin with a sharp point, which is necessary for easy entry of the cannula into the spinal canal (Figure 1). Induction of anesthesia was performed by intravenous injection of ketamine $10 \%(5 \mathrm{mg} / \mathrm{kg})$ and diazepam $(0.28 \mathrm{mg} / \mathrm{kg})$ and isoflurane gas was used to maintain anesthesia. The lumbosacral junction gap can be found either by counting the lumbar vertebras from cranial to caudal up to the last lumbar vertebrae (L7) or by palpating iliac wing tuberosity and drawing a line which crosses them perpendicular to the longitudinal axis of spine (Figure 2a). A $2 \mathrm{~mm}$ incision was made on the skin with a surgical blade to facilitate cannula entry and prevent damage to the skin at the entry site. The cannula and mandarin enter the spinal canal at a 60 -degree angle to the longitudinal axis of the spine, and then the mandarin is removed (Figure 2b). While passing the cannula into the spinal canal, penetration through the dorsal surface ligaments is sensible by the performer.

A blunt radiopaque wire designed for this purpose is passed through the cannula to confirm its correct placement in the canal (Figure 2c). Afterward Topogram and CT scan were performed for confirmation (Figure 2d).

\section{Behavioral Evaluation}

Twenty-four hours, and 7 and 28 days after the cannula placement in the lumbosacral junction and entrance in the epidural space, all the cats were evaluated neurologically, as follows:

1. Ability to bear weight with balance on the hind limbs/ Inability in weight-bearing on the hind limbs

2. Normal gait/imbalance and inability to gait normally

3. Ability to voluntarily urinate/urinary retention

4. Positive anal reflex/Negative anal reflex

\section{MR Imaging}

MRI and diffusion tensor imaging (DTI) imaging was performed on days 7 and 28 after cannula placement by the MRI scanner (Siemens Magnetom Prisma 3T, Germany) using a 32-channel spine coil. All cats were placed in a supine position under deep anesthesia (intramuscular injection of ketamine $10 \%$ (5 mg $/ \mathrm{kg}$ ) and medetomidine (10 to $40 \mu \mathrm{g} / \mathrm{kg})$ ). To avoid spine deviation in anesthetized cats, a restraining device was designed that could be placed in the magnetic space of the MRI scanner. Lumbosacral region was considered for MRI and DTI imaging in all cats. Imaging with $\mathrm{T} 1 \mathrm{~W}$ and $\mathrm{T} 2 \mathrm{~W}$ sequences (in sagittal and transverse views), STIR, and also DTI imaging was performed using the parameters recorded in Table 1 . The signal intensity index was evaluated for the T2-weighted and STIR sequences at the lumbosacral region, using e-film software (Workstation 3.4, 2010) to determine the severity of the possible injury. The DTI data were evaluated

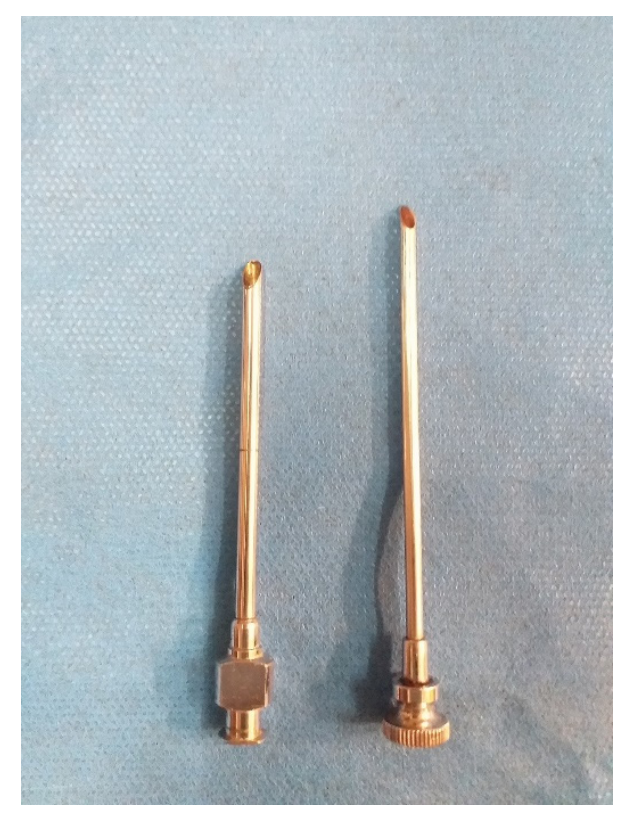

Figure 1. Stainless Steel Cannula. 


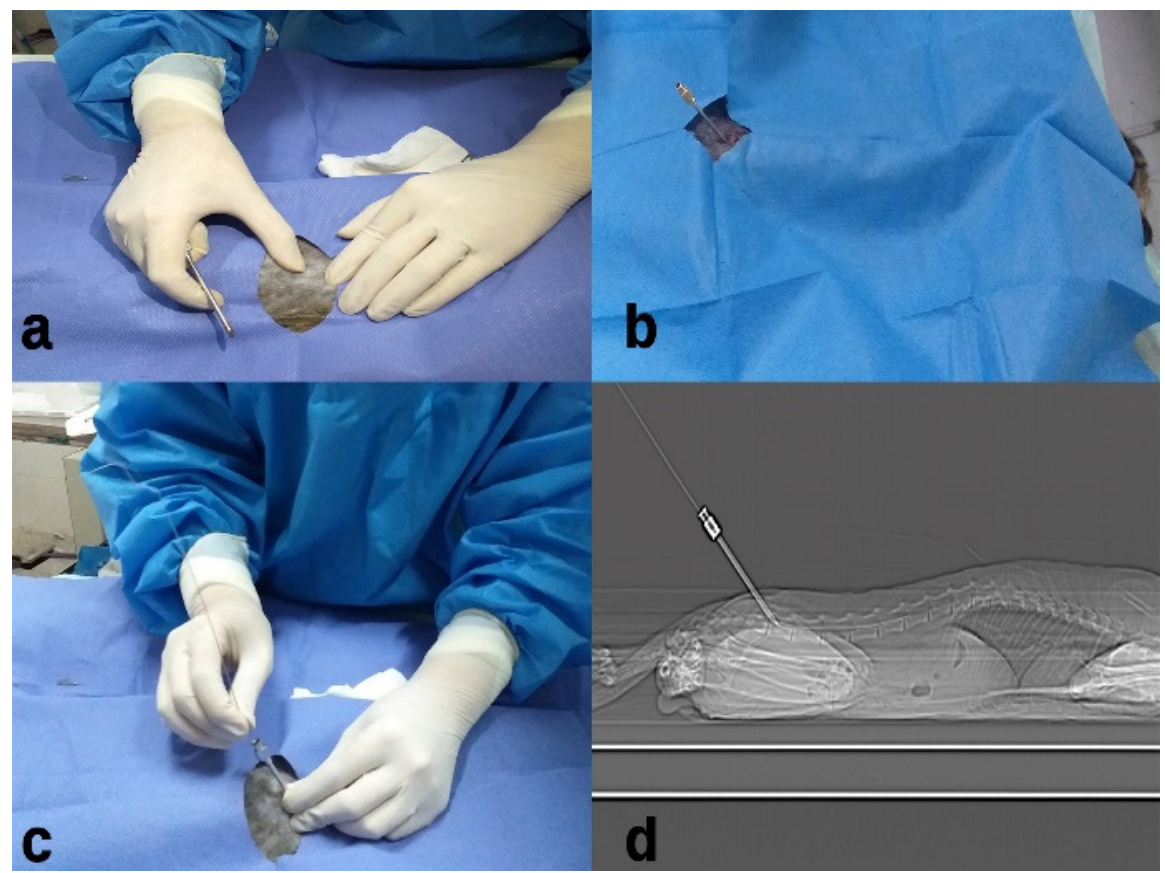

Figure 2. Cannula Insertion. (a) Palpation and detection of lumbosacral region, (b) cannula insertion in 60-degree angle according to vertebral column, (c) radiopaque blunt wire insertion due to correct placement of cannula confirmation, and (d) Final confirmation of correct cannula placement in vertebral canal by Topogram.

by data processing software Explore DTI V4.8.6. Three dimensional (3D) Tractography images were extracted for macroscopic evaluation of neural tracts.

\section{Results}

\section{General Observations}

All stages of anesthesia and cannula insertion were successfully performed in all studied cases. Correct cannula placement was confirmed in all cases by entering without resistance in wire passage and CT scan Topogram. No sign of active bleeding was observed at the site of insertion. CT scan images revealed no signs of bone damage at the vertebrae after cannula placement. During the study, the insertion site had no discharge or signs of infection in all subjects.

\section{Behavioral Evaluation}

After complete recovery, all cats had normal gait and kept balance on all four limbs. Also, there were no signs of injury to the cauda equina, such as urinary retention, tail dropping, lack of anal reflex, and so on. The behavioral assessment results in all cats on days 7 and 28 after the start of the study were similar to the first 24 hours.

\section{MRI Results}

The results of MRI evaluation 7 days after cannula

Table 1. DTI and MRI Parameters in T1-W, T2-W, and STIR Sequences

\begin{tabular}{|c|c|c|c|c|}
\hline Parameter & T2-Weighted Sequence & T1-Weighted Sequence & STIR Sequence & DTI \\
\hline Turbo factor & 23 & 4 & & $240 \mathrm{~mm}$ \\
\hline Matrix & & & & $128 \times 124$ \\
\hline Field of view & $220 \mathrm{~mm}$ & $220 \mathrm{~mm}$ & $220 \mathrm{~mm}$ & \\
\hline Slices & 9 & 9 & 32 & 12 \\
\hline Slices thickness & $3 \mathrm{~mm}$ & $3 \mathrm{~mm}$ & $2 \mathrm{~mm}$ & $2 \mathrm{~mm}$ \\
\hline Voxel size & $0.7 \times 0.7 \times 3 \mathrm{~mm}^{3}$ & $0.7 \times 0.7 \times 3 \mathrm{~mm}^{3}$ & $0.4 \times 0.4 \times 3 \mathrm{~mm}^{3}$ & $1.9 \times 1.9 \times 2 \mathrm{~mm}^{3}$ \\
\hline Repetition time (TR) & $3190.3 \mathrm{~ms}$ & $647.0 \mathrm{~ms}$ & $3170 \mathrm{~ms}$ & $10000 \mathrm{~ms}$ \\
\hline Echo time (TE) & $101.0 \mathrm{~ms}$ & $9.8 \mathrm{~ms}$ & $47 \mathrm{~ms}$ & $90.0 \mathrm{~ms}$ \\
\hline Flip angle & $150^{\circ}$ & $150^{\circ}$ & $150^{\circ}$ & \\
\hline B-value & & & & 0 and $800 \mathrm{~s} / \mathrm{mm}^{2}$ \\
\hline Phase-encoding direction & cranial-caudal & cranial-caudal & right-left & ventrodorsally \\
\hline
\end{tabular}


placement in all sequences did not show a significant change in signal intensity index in the lumbosacral spinal nerves and only the soft tissue reaction was evident around the entrance site (Figure $3 \mathrm{~b}$ ). MRI images 28 days after cannula placement also confirmed the initial imaging findings. Also, any abnormal changes in the cauda equina and nerve branches at the entry site were ruled out by tractography. Figure 3 a shows a tractography image of the spinal nerves in the lumbosacral region with normal continuity.

\section{Discussion}

So far, many studies have been conducted to achieve a standard spinal cord injury model similar to human spinal cord injuries to help find an appropriate treatment. In this study, we introduced an approach to access the epidural space in the cat which is simple, causes minimal pathophysiological changes, and does not have the risk of complications such as bleeding and infection. This approach can be used in minimally invasive spinal cord injury modeling methods such as compression spinal cord injury using balloon catheters.

Laminectomy is a method in which part of the dorsal lamina of the vertebra is removed. This method is used for spinal cord injury treatment and reduces secondary damages. Laminectomy also damages surrounding tissues such as blood vessels and muscles, leading to scar tissue and fibrous adhesions formation. Also, spinal cord exposure can be associated with serious complications such as infection and spinal cord displacement. ${ }^{8}$

Over time, researchers have introduced approaches without the need for laminectomy to create models of spinal cord injury. Kuchner et al and Lim et al inserted the balloon catheter into the epidural space through a hole in the dorsal lamina. ${ }^{9,10}$ This method was also used by Yang and colleagues in a rabbit animal model which allowed spinal cord injuries to be induced in several different regions of the spinal cord simultaneously. ${ }^{11}$ Although no laminectomy was performed in mentioned procedures, damage to the surrounding muscle and bone following surgery for dorsal lamina exposure was inevitable.

Fukuda et al also used the lateral intervertebral foramen approach in dogs to access the spinal canal, but it should be noted that in their approach, access to the lateral intervertebral foramen is required through surgical intervention and separation of muscles and ligaments. ${ }^{12}$

To solve this problem, Purdy and colleagues used fluoroscopic guidance to access the lateral intervertebral foramina. Although the risk of infection decreased in this method, the risk of damage to the spinal nerves, the spinal branch arteries, and veins that pass through the lateral intervertebral foramina remained, which often resulted in severe bleeding., ${ }^{5,13}$

In the present study, as in the study by Lee and colleagues, the percutaneous approach without significant surgical incision was used. In this approach, the risk of infection is minimized, and also, the risk of vascular and neurological damage is lower than the method of Purdy and colleagues. The percutaneous approach to the lumbosacral region has recently been used in many studies, including studies by Yoon and colleagues in 2017 and 2018. They developed a closed spinal cord injury model that allowed them to accurately evaluate histopathological changes and compared them with changes in DTI findings. ${ }^{14,15}$ Lee

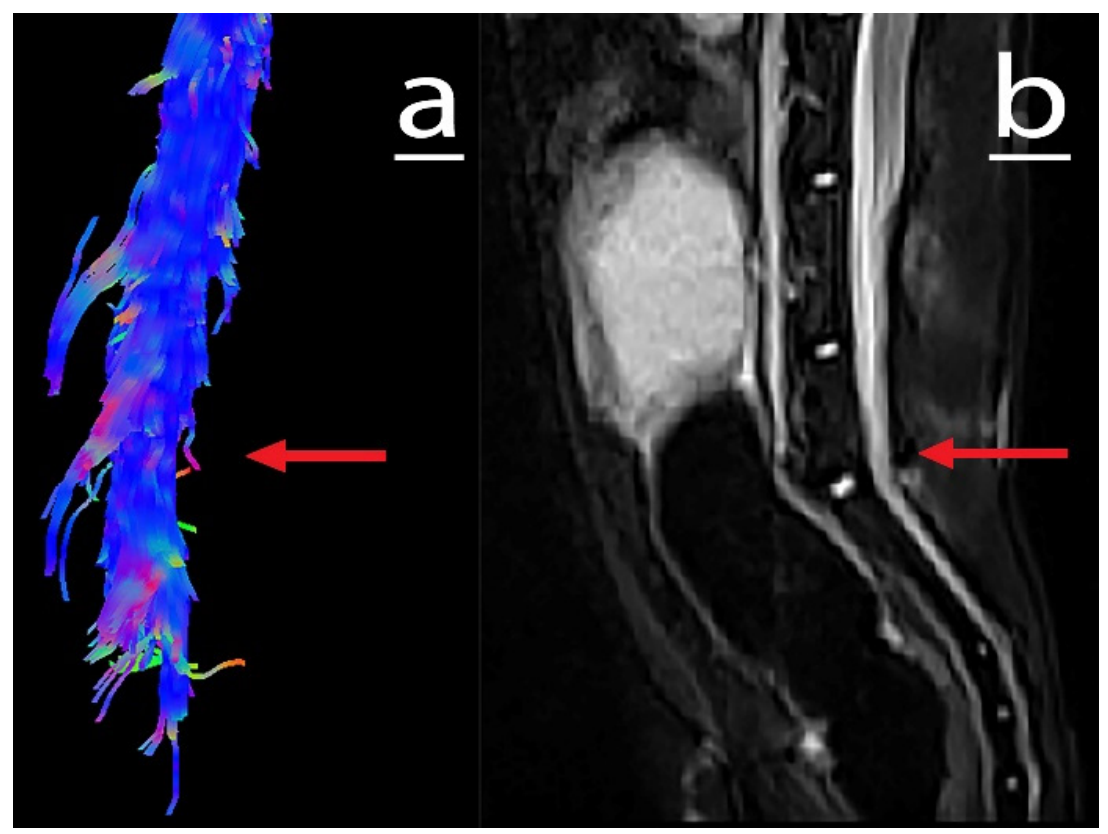

Figure 3. a) Spinal Cord Termination Tractography. Cannula insertion region pointed by red arrow, b) sagittal T2 weighted sequence of spinal cord termination. Cannula insertion region pointed by red arrow. 
and colleagues used fluoroscopic guidance for precise placement of the spinal needle and catheter. ${ }^{8}$ It should be noted that fluoroscopic guidance in small animals is not as practical as larger models, even in small dogs. For this reason, in Purdy and colleagues' study, dogs weighing 18$36 \mathrm{~kg}$ were used. ${ }^{5,13}$ Over the last three decades, cats, as spinal cord injury animal models, have been pioneers in the development of medical and surgical studies in this field. More advanced locomotor systems than rodents, more similar spinal cord to humans, and the possibility for easier and longer care than the monkey models are the advantages of using this animal model. ${ }^{16}$ However, the difficulty of fluoroscopy application in animals with smaller body sizes like cats, has limited using them for fluoroscopy dependent procedures. A challenge which was eliminated in this study, which allowed us to use the mentioned advantages of cat in spinal injury models without the need for fluoroscopy.

We introduced a simple and practical approach to access the epidural space. It should be noted that the cannula used in this study has an inner diameter of 3 $\mathrm{mm}$, which provides good access to the epidural space for the desired interventions, including the placement of a balloon catheter. This percutaneous approach does not require fluoroscopy and also does not risk damaging the spinal nerves and blood vessels in the lumbosacral region. This minimally invasive procedure prevents MRI imaging artifacts and error in the evaluation of the study, and does not cause any unwanted damage along the spinal cord. Moreover, it minimizes the risk of infection and increases the similarity of the condition to spinal cord injury in humans. Therefore, the pathophysiological changes after spinal cord injury and its treatment can be performed at a higher level of accuracy.

\section{Authors' Contribution}

AA designed and performed experiments, analyzed data and wrote the paper. MP supervised the research, Designed and performed experiments. MMD supervised the research. JS co-author. MM performed MRI data analysis. SE performed imaging part of experiment. RE analyzed data and wrote the paper

\section{Conflict of Interest Disclosures}

The authors declare that they have no conflict of interests.

\section{Ethical Statement}

This study was approved by Ethics Committee for Animal care of the Faculty of Veterinary Medicine, University of Tehran (Code: 328886.76).

\section{Funding}

There was no fund for this article

\section{References}

1. Hayta E, Elden H. Acute spinal cord injury: a review of pathophysiology and potential of non-steroidal antiinflammatory drugs for pharmacological intervention. J Chem Neuroanat. 2018;87:25-31. doi: 10.1016/j. jchemneu.2017.08.001.

2. Nardone R, Florea C, Höller Y, Brigo F, Versace V, Lochner $\mathrm{P}$, et al. Rodent, large animal and non-human primate models of spinal cord injury. Zoology (Jena). 2017;123:10114. doi: 10.1016/j.zool.2017.06.004.

3. Mattucci S, Speidel J, Liu J, Kwon BK, Tetzlaff W, Oxland TR. Basic biomechanics of spinal cord injury - how injuries happen in people and how animal models have informed our understanding. Clin Biomech (Bristol, Avon). 2019;64:58-68. doi: 10.1016/j.clinbiomech.2018.03.020.

4. Sharif-Alhoseini M, Khormali M, Rezaei M, Safdarian M, Hajighadery A, Khalatbari MM, et al. Animal models of spinal cord injury: a systematic review. Spinal Cord. 2017;55(8):714-21. doi: 10.1038/sc.2016.187.

5. Purdy PD, Duong RT, White CL 3rd, Baer DL, Reichard RR, Pride GL Jr, et al. Percutaneous translumbar spinal cord compression injury in a dog model that uses angioplasty balloons: MR imaging and histopathologic findings. AJNR Am J Neuroradiol. 2003;24(2):177-84.

6. Chung WH, Lee JH, Chung DJ, Yang WJ, Lee AJ, Choi CB, et al. Improved rat spinal cord injury model using spinal cord compression by percutaneous method. J Vet Sci. 2013;14(3):329-35. doi: 10.4142/jvs.2013.14.3.329.

7. Albus U. Guide for the Care and Use of Laboratory Animals. 8th ed. Washington, DC: The National Academies Press; 2012.

8. Lee JH, Choi CB, Chung DJ, Kang EH, Chang HS, Hwang $\mathrm{SH}$, et al. Development of an improved canine model of percutaneous spinal cord compression injury by balloon catheter. J Neurosci Methods. 2008;167(2):310-6. doi: 10.1016/j.jneumeth.2007.07.020.

9. Kuchner EF, Hansebout RR, Pappius HM. Effects of dexamethasone and of local hypothermia on early and late tissue electrolyte changes in experimental spinal cord injury. J Spinal Disord. 2000;13(5):391-8. doi: 10.1097/00002517200010000-00004.

10. Lim JH, Jung CS, Byeon YE, Kim WH, Yoon JH, Kang $\mathrm{KS}$, et al. Establishment of a canine spinal cord injury model induced by epidural balloon compression. J Vet Sci. 2007;8(1):89-94. doi: 10.4142/jvs.2007.8.1.89.

11. Yang C, Yu B, Ma F, Lu H, Huang J, You Q, et al. What is the optimal sequence of decompression for multilevel noncontinuous spinal cord compression injuries in rabbits? BMC Neurol. 2017;17(1):44. doi: 10.1186/s12883-0170824-3.

12. Fukuda S, Nakamura T, Kishigami Y, Endo K, Azuma T, Fujikawa $T$, et al. New canine spinal cord injury model free from laminectomy. Brain Res Brain Res Protoc. 2005;14(3):171-80. doi: 10.1016/j.brainresprot.2005.01.001.

13. Purdy PD, White CL 3rd, Baer DL, Frawley WH, Reichard RR, Pride GL Jr, et al. Percutaneous translumbar spinal cord compression injury in dogs from an angioplasty balloon: MR and histopathologic changes with balloon sizes and compression times. AJNR Am J Neuroradiol. 2004;25(8):1435-42.

14. Yoon H, Kim J, Moon WJ, Nahm SS, Zhao J, Kim HM, et 
al. Characterization of chronic axonal degeneration using diffusion tensor imaging in canine spinal cord injury: a quantitative analysis of diffusion tensor imaging parameters according to histopathological differences. J Neurotrauma. 2017;34(21):3041-50. doi: 10.1089/neu.2016.4886.

15. Yoon H, Moon WJ, Nahm SS, Kim J, Eom K. Diffusion tensor imaging of scarring, necrosis, and cavitation based on histopathological findings in dogs with chronic spinal cord injury: evaluation of multiple diffusion parameters and their correlations with histopathological findings. J Neurotrauma. 2018;35(12):1387-97. doi: 10.1089/ neu.2017.5409.

16. Shkorbatova PY, Lyakhovetskii VA, Merkulyeva NS, Veshchitskii AA, Bazhenova EY, Laurens J, et al. Prediction algorithm of the cat spinal segments lengths and positions in relation to the vertebrae. Anat Rec (Hoboken). 2019;302(9):1628-37. doi: 10.1002/ar.24054. 